\title{
DIFFERENTIAL HEBBIAN LEARNING
}

\author{
Bart Kosko \\ VERAC, Inc., 9605 Scranton Road, San Diego, CA 92121-1771
}

\section{ABSTRACT}

The differential Hebbian law $\dot{e}_{i j}=\dot{C}_{i} \cdot \dot{C}_{j}$ is examined as an alternative to the traditional Hebbian law $\dot{e}_{i j}=c_{i} c_{j}$ for updating edge connection strengths in neural networks. The motivation is that concurrent change, rather than just concurrent activation, more accurately captures the "concomitant variation" that is central to inductively inferred functional relationships. The resulting networks are characterized by a kinetic, rather than potential, energy. Yet we prove that both system energies are given by the same entropy-like functional of connection matrices, $\operatorname{Trace}(\dot{E} E)$. We prove that the differential Hebbian is equivalent to stochastic-process correlation (a cross-covariance kernel). We exactly solve the differential Hebbian law, interpret the sequence of edges as a stochastic process, and report that the edge process is a submartingale: the edges are expected to increase with time. The submartingale edges decompose into a martingale or unchanging process and an increasing or novelty process. Hence conditioned averages of edge residuals are encoded in learning though the network only "experiences" the unconditioned edge residuals.

\section{INTRODUCTION}

Synaptic connections are causal connections. Their modification is an act of inductive inference. Edge connection strengths are inferred from node (neuron, processing element, etc.) behavior. This suggests that the modification criteria should, at minimum, reflect the logico-causal c:iteria of scientific method used for attributing a functional relationsilp among variable quantities. And what are these criteria but that the quantities should move or change in the same or opposite directions aid that the "cause" temporally precedes the "effect?" Eighteenth century expiricist philosopher David Hume observed that we habitually make causal ascriptions when we observe sustained "constant conjunctions of events." In his System of Logic, nineteenth century empiricist philosopher John Stuart Mil1 ${ }^{2}$ refined Hume's observation. Mill observed that the causalit ${ }^{\prime}$ we attempt to inductively infer is simply the "concomitant variation" of the variable quantities, and this formulation, often restated in the jargon of statistical inference, remains the operative notion $c f$ causality today.

\section{THE DIFFERENTIAL HEBBIAN LAW}

The task is to specify the cynamical equation $\dot{\dot{s}}_{i j}=f(C, E)$ of the edge $e_{i j}$ that connects node (causai variuice) $c_{i}$ to $C_{j}$ in a network, where $C^{1}=\left(c_{1}, \ldots, c_{n}\right)$ is the node state vector and $E=\left[e_{i j}\right]$ is the matrix of edue connections. We assume the transfor cquation $\dot{C}_{i}=\Im(C, E)$ is given for each node (and is dominated by an inner product of input edges and 
nodes : $\quad \dot{\mathrm{C}}_{\mathrm{i}}=\sum_{\mathrm{k}} \mathrm{C}_{\mathrm{k}} \mathrm{e}_{\mathrm{ki}}+\mathrm{D}_{\mathrm{i}}$,

where $D_{i}$ contains all other terms. This functional form, a sort of neural OR gate, predominates in neural modeling. We assume that the righthand side of (1) contains terms internal and external to the network. The simplest internal terms are the inner product minus the current activation $C_{i}$. The external terms are an observation or sensor term $\mathrm{O}_{i}$ and an advice or teacher or expert-response term $\mathrm{R}_{i}$, both of arbitrary structure. Hence $D_{i}=O_{i}+R_{i}-C_{i}$.

The standard selection of $f$ in $\dot{e}_{i j}=f(C, E)$ is attributed to the "correlation learning" hypothesis of Hebb" ${ }^{3}$, which is simply that concurrent activation of nodes increases the "synaptic efficacy" or strength of the connection between them. In a nutshell,

$$
\dot{e}_{i j}=C_{i} c_{j} \cdot
$$

Typically the current strength $e_{i j}$ is subtracted from the righthand side to represent "forgetting" or "memory decay" (or to slow the otherwise exponential growth?). This linear appendage does not affect the current analysis and for notational simplicity we omit it.

Equation (2) is widespread in the neural net literature. It accurs in the famous Grossberg ${ }^{4-6}$ equations and the related equations of Hopfield ${ }^{7}$ and in similar form in the adaptive equations of most neural modelers. But apart from referencing Hebb's (nonmathematical) conjecture, it seems the operative argument for using (2) is simply that everyone uses it. For surely the problems with (2) warrant investigating alternatives. To begin, (2) promotes spurious causal associations. If any two processors or nodes are active in a network, no matter how big the network, how far apart the nodes, or how independent their patterns of activation, the Hebbian law (2) grows a causal connection between them. Concomitant activation replaces concomitant variation. Worse, the spurious causal attributions tend to grow exponentially fast (as can be seen from the exponential form of the exact solution of (2) when the forget term $-e_{i j}$ is appended). In practice this necessitates "hardclipping" of interconnects both during training and classification sessions. Finally, transfer functions must first be integrated before (analytically) including them in (2). This integration is never easy.

A natural alternative to (2) is the differential Hebbian law:

$$
\dot{\mathrm{e}}_{i j}=\dot{\mathrm{C}}_{i} \dot{\mathrm{C}}_{j} \text {. }
$$

The cifferential Hebbian measures concomitant variation. It imputes causality according to (lagged) conjunctions of event changes. As a result, it truly behaves in correlation fashion. For although the functions $c_{i}$ are nonnegative, their derivatives are not. Hence the connection $e_{i j}$ strengthens iff both nodes agree in sign, hence iff both nodes move in the same direction. (Note this implies concurrent activation.) Negative causality accumulates if they move in opposite directions. Moreover, transfer functions such as (1) can be directly plugged 
into (3), allowing many properties to be determined analytically. Below we exploit this fact to solve the system (1) and (3) for $e_{i j}(t)$.

The idea of using rates of change in learning laws is spreading. Two especially noteworthy cases are the drive-reinforcement model of Klopf ${ }^{8-9}$ and the backward-error-propagation model of Rumelhart- -4 , Hinton, and Williams. Klopf reports that a wide array of Pavlov-like learning behaviors is accurately predicted (retrodicted) by a changebased law. Rumelhart uses a time derivative of input activation (essentially $\dot{C}_{\dot{j}}$ ) in his "generalized delta law" (subsuming the classical perceptron convergence theorem) to solve the exclusive-or problem, the parity problem, and a variety of others.

\section{KINETIC ENERGY CONNECTIONS}

The energy of a network is the sum of the eigenvalues of the product connection matrix $E \dot{E}$. Here $\dot{E}$ is the $n$-by-n symmetric matrix of connection changes $\left[\dot{e}_{i j}\right]$. For Hopfield networks, Abu-Mostafa and St. Jaques ${ }^{11}$ have shown that the number of energy minima (memory sites) is no more than $\mathrm{n}$. We conjecture that these and comparable equilibria correspond to the eigenvalues of $\mathrm{EE}$.

To prove the eigenvalue theorem, define the pottential energy (P.E.) and kinetic energy (K.E.) of the network [C, C, E, E] as follows:

$$
\begin{aligned}
& \text { P.E. }=C^{\mathrm{T} E C}=\sum_{i} \sum_{j} \mathrm{C}_{i} \mathrm{C}_{j} \mathrm{e}_{i j}, \\
& \text { K.E. }=\dot{\mathrm{C}}^{\mathrm{T}} \mathrm{E} \dot{\mathrm{C}}=\sum_{i} \sum_{j} \dot{\mathrm{C}}_{i} \dot{\mathrm{C}}_{j} e_{i j} .
\end{aligned}
$$

The trick is that if the Hebbian law (2) is in force, then $\dot{e}_{i j}$ replaces $c_{i} c_{j}$ in (4). If the differential Hebbian law (3) is in force, then $\dot{\mathrm{e}}_{i j}$ replaces $\dot{\mathrm{C}}_{i} \dot{\mathrm{C}}_{j}$ in (5). Hence then P.E. = K.E. !

THEOREM. $\quad \sum_{i=1}^{n} \lambda_{i}=\left\{\begin{array}{ll}\text { P.E. } & \text { if } \dot{E}=c \mathrm{C}^{\mathrm{T}} \\ \text { K.E. } & \text { if } \dot{\mathrm{E}}=\dot{\mathrm{C}} \dot{\mathrm{C}}^{\mathrm{T}}\end{array}\right.$,

where $\lambda_{1}, \ldots, \lambda_{n}$ are the eigenvalues of $\dot{E} \dot{E}(\dot{E} E)$.

PROOF. By basic linear algebra, the sum of eigenvalues of $\dot{E E}$ equals the sum of diagonal elements, the trace Trace(EE). Then

$\operatorname{Trace}(\dot{E})=\sum_{i}(\dot{E E})_{i i}=\sum_{i} \sum_{j} e_{i j} \dot{e}_{j i}=\sum_{I} \sum_{j} e_{i j} \dot{e}_{i j}$

equals P.E. or K.E. according as $\dot{\mathrm{E}}$ equals $\mathrm{C} \mathrm{C}^{\mathrm{T}}$ or $\dot{\mathrm{C}} \dot{\mathrm{C}}^{\mathrm{T}}$. Q.E.D.

Two comments are in order. First, when a no weight-change analysis is desired--as it often is in Grossberg and Hopfield networks--simply invor the Hebbian or differential Hebbian law to replace $\dot{e}_{i j}$ with $C_{i} C_{j}$ or $C_{i} C_{j}$. Second, Trace(EE) can be viewed as an entropy-like functional. For, in 
learning networks, the edges or neural pathways adapt slowly to the activation or signals flowing through them. I.e., $\dot{e}_{i j}$ tends to be smaller than $e_{j j}$ at any time $t$. Let us model this property with the hypothesis that $\dot{e}_{i j} \cong$ In $e_{i j}$. Now recall that Von Neumann ${ }^{12}$ defined the entropy of a (quantum-mechanical) system with Trace( $P$ InP), where $P$ is a positive semidefinite matrix with Trace $(P)=1$, thus generalizing the log-of-probability entropy of Boltzmann and others (including Shannon). Watanabe ${ }^{13}$ has kept this entropy measure current by showing how its minimization corresponds to. pattern recognition in many cases. On our learning hypothesis, Trace $(E E) \cong$ Trace $(P$ InP) for suitable $P$. However, since Trace $(E)=\operatorname{Trace}(E)=0, E$ cannot be normalized to 1 and thus an exact identity between the two trace functionals cannot be expected to hold.

\section{CORRELATION AND AN EXACT SOLUTION}

Let us interpret the node vector $\mathrm{C}$ as a random vector. Let each node $C_{i}$ be a stochastic process: $C_{i}: T \times \Omega \rightarrow[0, \infty)$, where $T$ is an index set of time values and $(\Omega, A, P)$ is the probability space. Suppose we know the present value of $C_{i}(t)\left(C_{j}(t, \omega)\right)$. Then given no other information, and being true scientific empiricists, what is our best prediction of $C_{i}(t+1)$ ? Surely it is just the present value $C_{i}(t)$. Let us call this the quasi-martingale assumption:

$$
E_{P}\left(C_{i}(t+1)\right)=C_{i}(t),
$$

where $\mathrm{E}_{\mathrm{P}}$ is the expectation with respect to probability measure $\mathrm{P}$. If we now use the discrete differential Hebbian, we arrive at

$$
\begin{aligned}
e_{i j}(t+1) & =e_{i j}(t)+\Delta c_{i}(t) \Delta c_{j}(t+1) \\
& =\sum_{s}^{t} \Delta c_{i}(s) \Delta C_{j}(s+1) \\
& =\sum_{s=0}^{t}\left[C_{i}(s)-E\left(C_{i}(s)\right)\right]\left[C_{j}(s+1)-E\left(C_{j}(s+1)\right)\right],
\end{aligned}
$$

which has the form of a nonnormalized cross-covariance kernel--the key term in the definition of statistical correlation. This identity establishes a direct connection with correlation and our operative definition of causality, the differential Hebbian (3).

Since the differential Hebbian uses derivatives of transfer functions, we can plug in dynamical equations such as (1) and.solve directly for $e_{i j}$. We demonstrate that the network [C, C, E, E] given by (1) and (3) can be easily solved. The trick is that $e_{i j}$ can be pulled out of the inner product in (1). (3) then takes the form $\dot{e}_{i j}+a e_{i j}=b-a$ first-order inhomogeneous ordinary differential equation with variable coefficients, one of the most tractable differential equations. Details of this expansion can be found in Kosko14-15. Here we state the exact solution: 
$e_{i j}(t)=e^{\int_{0}^{t} p(s) d s}\left(K+\int_{0}^{t} q(s) e^{-\int_{0}^{s} p(u) d u} d s\right)$,

where the functions $p$ and $q$ contain the grouped terms that occur in the manipulation when $\dot{C}_{i}$ and $\dot{C}_{j}$ are expanded with (1), which for brevity we omit. The essential point is that $p$ only contains the observation term $O_{i}$ and contains it linearly and $q$ contains both $o_{i}$ and $o_{j}$ and the product $o_{i} o_{j}$. The constant $k$ is given by $k=e_{i j}(0)$.

\section{EDGES AS SUBMARTINGALES}

The edge $e_{i j}$ is expected to increase in strength in time as information accumulates under the differential Hebbian hypothesis (3). This answers the question where edges tend on average, or, put another way, how connected networks become on average. To formalize such speculations, we interpret $e_{i j}$ as stochastic process-- $e_{i j}: T \times \Omega \rightarrow(-\infty, \infty)-$ on the probability space $(\Omega, A, P)$. We superscript with $t$ to index the random variables $\left\{\mathrm{e}_{\mathrm{ij}}^{\mathrm{t}}\right\}$.

External input enters the network through the observation terms $O_{i}$ in (1). A hearty scientific empiricism dictates that, fundamentaly, we know nothing of the future flux of experience. Experimentation is the resultant coping device. What does this mean for future values of the random variable $o_{i}$ when we know the present value $o_{i}^{s} s<t$ ? More precisely, suppose we have an increasing sequence of sets of information in the form of a "filtration" of sigma-algebras $A_{s} \subset A_{t} \subset A_{,} s \leq t$. Then what is the conditional expectation $E\left(O_{i}^{t} \mid A_{S}\right)$ ? Surely the most we can assert is the present observed value. This constitutes a network martingale assumption:

$$
E\left(O_{i}^{t} \mid A_{s}\right)=O_{i}^{S} \text { if } s \leq t .
$$

Since the product $o_{i} o_{j}$ occurs in the q term of solution (9), we must further assume that the two observation plocesses are conditionally independent martingale processes: $E\left(O_{i} O_{j} \mid A_{s}\right)=O_{i}^{S} O_{j}^{S}$ for $s \leq t$. (Technically we also assume the processes are suitably integrable and filtration measurable.)

So is the edge process a martingale if the observation processes are conditionally independent martingales? It turns out that the edge process is a submartingale process. (The proof, in Koskol4, uses Jensen's inequality and the convexity of the exponentials in the modified (9).) The expected future value is at least as big as the present value when conditioned on all the information available up to the present time. Hence $E\left(e_{i j} \mid A_{s}\right) \geq e_{i j}^{s}$ for $s \leq t$. The proof is facilitated by interpreting the integrals in solution (9) as conditional expectations (since stochastic integrals are always martingales). For instance, the first integral then takes the form $E\left(p_{t} \mid A_{t}\right)$. This interpretation keeps account of the acquired information and outputs functions not constants. 
Hence neural or causal networks can be expected to become more connected on average as time passes. The causal consequences of this may seem anti-entropic, perhaps even a violation of the second law of thermodynamics. But in fact total connectivity is the maximum entropy case (where entropy is intuitively interpreted). Order is established by a contrast between edge connections and edge disconnections, as in a military hierarchy or a bureaucratic dictatorship rather than in a voting democracy. Better, in terms of dynamic communication connections, note how a monitored debate tends unidirectionally to a free-forall discussion.

Another consequence is that edge processes can be decomposed into a sum of a martingale and an increasing process:

$$
e_{i j}^{t}=M_{i j}^{t}+N_{i j}^{t}
$$

where $\mathrm{M}$ is the martingale process and $\mathrm{N}$ is either positive or zero. $\mathrm{M}$ represents what stays the same in the connection process. Hence $N$ represents what is new or novel, and, in the spirit of Kohonen ${ }^{16}$, we call it the novelty process. A consequence of the Doob-Meyer decomposition (11) is that $N$, and hence its residual, can be written as follows:

$$
\begin{aligned}
& N_{i j}^{t}=\sum_{k}^{t} E\left(e_{i j}^{k}-e_{i j}^{k-1} \mid A_{k-1}\right), \\
& N_{i j}^{t}-N_{i j}^{t-1}=E\left(e_{i j}^{t}-e_{i j}^{t-1} \mid A_{t-1}\right) .
\end{aligned}
$$

of the many things that can be said of this novelty process, perhaps the most significant concerns what it, and hence the edge process, encodes. (13) makes the contribution to differential Hebbian learning. What is significant is that a conditioned average is encoded even though not experienced. The expected edge residual is conditioned on exactly what it should be--all the information accumulated up to the present.

\section{REFERENCES}

1. D. Hume, An Inquiry Concerning Human Understanding (1748).

2. J. S. Mi11, A System of Logic (1843).

3. D. O. Hebb, The Organization of Behavior (1949).

4. S. Grossberg, Psvch. Rev., 58, 1 (1980).

5. S. Grossberg, Studies of Mind and Brain (Reidel, Boston, 1982).

6. M. A. Cohen and S. Grossberg, IEEE Trans. SMC, 13, 815 (1983).

7. J. J. Hopfield, Proc. Nat. Acad. Sci, 79, 2554 (1982).

8. A. H. Klopf, Proc. 2nd Conf on Comp. with Neural Net. (1986).

9. R. S. Sutton and A. G. Barto, Psych. Rev., 88, 135 (1981).

10. D. E. Rumelhart, G. E. Hinton, R. J. Williams, in Parallel Distributed Processing, Rumelhart and McClelland Eds. (MIT Press, 1986).

11. Y. S. Abu-Mostafa and J. St. Jacques, IEEE Trans. IT, 31, 461 (1985).

12. J. Von Neumann, Mathematical Foundations of Quantum Mechanics (Springer, Berlin, 1932).

13. S. Watanabe, Pattern Recognition: Human and Mechanical (Wiley, NY, 1985).

14. B. Kosko, "Adaptive Inference," subitted for publication (1986).

15. B. Kosko and J. S. Limm, Proc. SPIE, 579, 104 (1985).

16. T. Kohonen, Self-Organization and Associative Memory (SpringerVerlag, Berlin, 1984). 